\title{
Características químicas de substratos formulados com lodo de esgoto para produção de mudas florestais
}

\author{
Fernando E. V. Santos ${ }^{1}$, Sustanis H. Kunz ${ }^{2}$, Marcos V. W. Caldeira ${ }^{3}$, \\ Carlos H. S. Azevedo ${ }^{4} \&$ Otacilio J. P. Rangel ${ }^{5}$ \\ ${ }^{1}$ Programa de Pós-Graduação em Ciências Florestais/UFES, Jerônimo Monteiro, ES. E-mail: fernandoelair@gmail.com
${ }^{2}$ Departamento de Ciências Florestais e da Madeira/UFES, Jerônimo Monteiro, ES. E-mail: sustanis.kunz@ufes.br (Autor correspondente)
${ }^{3}$ Departamento de Ciências Florestais e da Madeira/UFES, Jerônimo Monteiro, ES. E-mail: mvwcaldeira@gmail.com
${ }^{4}$ Programa de Pós-Graduação em Ciências Florestais/UFES, Jerônimo Monteiro, ES. E-mail: carlosh_sa@hotmail.com
${ }^{5}$ Instituto Federal de Educação, Ciência e Tecnologia do Espírito Santo/IFES, Campus de Alegre, ES. E-mail: otaciliorangel@gmail.com
}

Palavras-chave: fertilidade dos substratos nutrição para plantas biossólido resíduos orgânicos

\begin{abstract}
R E S U M O
Objetivou-se, com o trabalho, caracterizar as propriedades químicas e as composições dos substratos formulados à base de lodo de esgoto em diferentes combinações com resíduos orgânicos e vermiculita, a fim de se obter um meio de crescimento adequado para o crescimento de mudas florestais. Foi realizada análise de teores totais e disponíveis dos nutrientes dos 26 substratos formulados a partir da mistura de lodo de esgoto com vermiculita e os resíduos fibra de coco, palha de café in natura, composto orgânico, casca de arroz carbonizada e casca de arroz in natura, nas proporções de 100:0, 80:20, 60:40, 40:60 e 20:80 (lodo de esgoto:materiais) e uma testemunha, constituída por substrato comercial. O substrato comercial proporcionou as maiores médias para os teores disponíveis de fósforo, cálcio e magnésio. $\mathrm{O}$ lodo de esgoto proporcionou aumento da fertilidade dos substratos com aumento de teores de nutrientes, sobretudo fósforo, nitrogênio e cálcio. Os substratos formulados com lodo de esgoto e composto orgânico, independente de suas proporções, foram considerados os mais adequados para a produção de mudas de espécies florestais.
\end{abstract}

Key words: substrate fertility plant nutrition biosolids organic waste

\section{Chemical characteristics of substrates formulated with sewage sludge for production of seedlings of forest species}

\begin{abstract}
A B S T R A C T
The objective of this study was to characterize the chemical properties and compositions of substrates formulated with sewage sludge in different combinations. The substrates were produced using organic waste and vermiculite, in order to obtain an adequate growth medium for the forest seedling. An analysis was made of the total and the available nutrient content on the 26 substrates, made with sewage sludge and vermiculite and waste from coconut fiber, in natura coffee straw, organic compost, carbonized rice husk and in natura rice husk, using the following ratios: 100:0, 80:20, 60:40, 40:60 and 20:80 (sewage sludge:material), and also a control consisting of commercial substrate. The commercial substrate provided the highest means for available levels of phosphorus, calcium and magnesium. The sewage sludge provided an increase of fertility of the substrates, with increased nutrient levels, mainly phosphorus, nitrogen and calcium. The substrates formulated with sewage sludge and organic compost, regardless of their ratios, were considered the most adequate for the production of seedling of forest species.
\end{abstract}

\section{INTRODUÇÃo}

No passado, o solo era utilizado como o principal substrato para a produção de mudas o qual, por sua vez, é o resultado da interação entre processos físicos, químicos e biológicos na rocha matriz. Novas tecnologias foram sendo aplicadas para garantir maior qualidade na produção de mudas; com isto, o solo foi perdendo seu espaço para substratos alternativos, como lodo de esgoto, casca de arroz carbonizada e in natura, esterco animal, vermicomposto e fibra de coco (Caldeira et al., 2008; Saidelles et al., 2009; Trazzi et al., 2012).
As características químicas dos substratos estão relacionadas à sua capacidade de fornecer nutrientes às plantas. A caracterização química dos substratos e de suas matériasprimas é fundamental para o conhecimento da formulação, recomendação e monitoramento de adubações, o que contribui para a qualidade dos substratos.

As características e os componentes dos substratos são muito variáveis. Dentre as características químicas aquelas que têm destaque são o potencial hidrogêniônico $(\mathrm{pH})$, a capacidade de troca de cátions (CTC), a salinidade e o teor de matéria orgânica (Schmitz et al., 2002; Kämpf, 2005). 
As propriedades químicas do substrato podem ser alteradas, ao contrário das propriedades físicas, que dificilmente podem ser modificadas após plantio da muda. Um substrato pode ser melhorado com o fornecimento de nutrientes providos ou não de fontes minerais e seu pH corrigido (Cabrera, 1999). De acordo com Bellote \& Dedecek (2006) a expressão dos efeitos das propriedades químicas dos substratos é reduzida por algumas situações, como deficiência hídrica, compactação e impedimentos físicos; já Wendling et al. (2007) e Kratz et al. (2013) verificaram, analisando as caracerísticas de substratos renováveis, que as propriedades físicas não interferiram nas características nutricionais.

Alguns estudos demonstraram que substratos formulados com o lodo de esgoto podem trazer benefícios à produção de mudas de espécies florestais haja vista que este resíduo contribui no fornecimento de nutrientes às mudas, além de ter sua contribuição para minimizar a poluição ambiental devido ao destino adequado do material. Trigueiro \& Guerrini (2003) por exemplo, avaliaram a viabilidade do uso de lodo de esgoto como componente do substrato para a produção de mudas de eucalipto e os resultados das análises químicas indicaram que o lodo de esgoto contém altos teores de nutrientes, com destaque para nitrogênio e fósforo. Guerrini \& Trigueiro (2004) avaliaram as características químicas de substratos com diferentes doses de lodo de esgoto e de casca de arroz carbonizada e verificaram que a quantidade de nutrientes no substrato aumentou com a elevação da percentagem de lodo de esgoto.

Wendling et al. (2007) avaliaram as características químicas $\mathrm{N}$ total, $\mathrm{P}, \mathrm{K}, \mathrm{Ca}, \mathrm{Mg}$ e $\mathrm{pH}$ de substratos para produção de mudas de Ilex paraguariensis St. Hil. e verificaram que tais características não interferiram nas variáveis de sobrevivência nem no crescimento das mudas.

A utilização de resíduos como componentes de substratos para a produção florestal pode ser uma alternativa viável para destinação final desses materiais porém é necessário que seja realizada a caracterização química dos resíduos para garantir a sobrevivência e o crescimento das mudas de espécies florestais. Assim, este trabalho teve como objetivo caracterizar as propriedades químicas e a composição dos substratos formulados à base de lodo de esgoto em diferentes combinações com outros resíduos orgânicos e vermiculita, a fim de se obter um meio de crescimento adequado para mudas de espécies florestais.

\section{MATERIAL E Métodos}

Associados ao lodo de esgoto, os componentes utilizados para a composição dos substratos foram fibra de coco (FC), vermiculita (VER), palha de café in natura (PCN), composto orgânico (CO), casca de arroz carbonizada (CAC), casca de arroz in natura (CAN) e substrato comercial (SC).

O lodo de esgoto (LE) utilizado proveio da estação de tratamento de esgoto Foz do Brasil S.A., situada no distrito de Pacotuba, município de Cachoeiro do Itapemirim, ES. O tratamento resultou do lodo, por meio do filtro anaeróbio em que as concentrações dos compostos químicos e características deste material (Tabela 1) foram fornecidas pela empresa utilizando a seguinte metodologia de análise: fósforo total: digestão com ácido nítrico + ácido sulfúrico; enxofre: análise gravimétrica com bário; nitrogênio total: Kjeldahl; Sólidos Totais: POP PA 058 Rev.02; Metais:(ICP-OES) Determinação: SMWW 3120 B / Preparo: EPA 3010A:1992; carbono orgânico: POP PA 182; pH: POP PA 056 / USEPA 9045 D. Para a utilização do lodo de esgoto na agricultura os teores dos elementos devem estar dentro dos níveis estipulados pela Resolução no 375, de 26 de Agosto de 2006 do Conselho Nacional do Meio Ambiente (Brasil, 2006).

Para a composição dos substratos, o lodo de esgoto foi mantido exposto a pleno sol ao ar livre em área experimental no viveiro da UFES durante 60 dias, longe do contato de humanos e animais, sendo periodicamente revolvido; posteriormente, o resíduo foi peneirado com malha de $2 \mathrm{~mm}$ (9 mesh) para homogeneização das partículas.

O composto orgânico foi produzido no viveiro com a utilização de esterco bovino proveniente das atividades pecuárias da Área Experimental I/Centro Ciências Agrárias da Universidade Federal do Espírito Santo (CCA/UFES) e de palha de café in natura doada por produtores rurais da região do Parque Nacional do Caparaó, ES. O mesmo volume de palha de café in natura e o esterco bovino foram misturados e permaneceram 60 dias em ambiente aberto para sua estabilização biológica; após este processo o material foi peneirado com malha de $2 \mathrm{~mm}$ para homogeneização.

Como tratamento testemunha utilizou-se o substrato comercial (SC) formulado com as seguintes matérias-primas: casca de pinus sp., esterco, serragem, fibra de coco, vermiculita, gesso agrícola, carbonato de cálcio, magnésio e aditivos (fertilizantes).

A fibra de coco (FC), vermiculita (VER), casca de arroz carbonizada (CAC) e casca de arroz in natura (CAN) foram provenientes de doação da empresa Fibria.

Tabela 1. Análise química do lodo de esgoto proveniente da Estação de Tratamento de Esgoto Foz do Brasil de Pacotuba distrito de Cachoeiro de Itapemirim, ES

\begin{tabular}{|c|c|c|}
\hline Parâmetros & $\begin{array}{l}\text { Resultados } \\
\text { analíticos }^{1}\end{array}$ & $\begin{array}{c}\text { CONAMA } 375(2006)^{2} \\
\left(\mathrm{mg} \mathrm{kg}^{-1}\right)\end{array}$ \\
\hline Arsênio & $\star<0,5 \mathrm{mg} \mathrm{dm}^{-3}$ & 41 \\
\hline Bário & $156 \mathrm{mg} \mathrm{dm}^{-3}$ & 1300 \\
\hline Cádmio & $<0,053 \mathrm{mg} \mathrm{dm}^{-3}$ & 39 \\
\hline Chumbo & $29 \mathrm{mg} \mathrm{dm}^{-3}$ & 300 \\
\hline Cobre & $98 \mathrm{mg} \mathrm{dm}^{-3}$ & 1500 \\
\hline Cromo & $26 \mathrm{mg} \mathrm{dm}^{-3}$ & 1000 \\
\hline Molibdênio & $3,5 \mathrm{mg} \mathrm{dm}^{-3}$ & 50 \\
\hline Níquel & $11 \mathrm{mg} \mathrm{dm}^{-3}$ & 420 \\
\hline Selênio & $<0,5 \mathrm{mg} \mathrm{dm}^{-3}$ & 100 \\
\hline Zinco & $409 \mathrm{mg} \mathrm{dm}^{-3}$ & 2800 \\
\hline Fósforo total & $4128 \mathrm{mg} \mathrm{dm}^{-3}$ & - \\
\hline pH (Suspensão a 5\%) & $5,2 \mathrm{mg} \mathrm{dm}^{-3}$ & - \\
\hline Enxofre & $1,3 \%$ & - \\
\hline Nitrogênio total Kjeldahl & $5646 \mathrm{mg} \mathrm{dm}^{-3}$ & - \\
\hline Nitrogênio amoniacal & $60 \mathrm{mg} \mathrm{dm}^{-3}$ & - \\
\hline Carbono orgânico total & $16 \%$ & - \\
\hline Potássio & $1623 \mathrm{mg} \mathrm{dm}^{-3}$ & - \\
\hline Sódio & $399 \mathrm{mg} \mathrm{dm}^{-3}$ & - \\
\hline Umidade & $15,3 \%$ & \\
\hline
\end{tabular}


Os substratos foram obtidos misturando-se, individualmente, o lodo de esgoto com os resíduos fibra de coco, palha de café in natura, casca de arroz in natura, casca de arroz carbonizada e composto orgânico, além da vermiculita, de acordo com as proporções em volume de100:0, 80:20, 60:40, 40:60 e 20:80 (lodo de esgoto:resíduos, vermiculita) e substrato comercial, totalizando 26 tratamentos.

Com base na carência de um padrão de realização de análises químicas de substratos, optou-se por fazer a análise química de rotina na determinação dos teores disponíveis e análise de tecido para determinação dos teores de nutrientes totais visto que, com exceção da vermiculita, os demais componentes dos substratos são orgânicos.

Os substratos foram submetidos às análises químicas no Laboratório de Recursos Hídricos do Departamento de Ciências Florestais e da Madeira/CCA/UFES. As análises dos substratos foram realizadas após secagem do material ao ar e peneiradas em malha de $2 \mathrm{~mm}$. Foram determinados os teores dos seguintes nutrientes: N, P, K, Ca, Mg, S, Fe, Zn, Cu, Mn, B. A determinação de $\mathrm{N}$ foi por digestão sulfúrica e destilação (Kjeldhal) e P, K, Ca, $\mathrm{Mg}, \mathrm{S}, \mathrm{Fe}, \mathrm{Zn}, \mathrm{Cu}$ e Mn por digestão nitroperclórica e o teor B obtido por digestão secada em mufla utilizando-se o método realizado conforme EMBRAPA (2009).

Foram analisados os teores disponíveis de fósforo, potássio, cálcio, magnésio, $\mathrm{pH}$, alumínio trocável ( $\mathrm{Al})$, acidez trocável $(\mathrm{H}+\mathrm{Al})$, capacidade de troca de cátions a $\mathrm{pH} 7$ (CTC a pH 7), soma de bases (SB), saturação por bases (V) e saturação por alumínio (m) de acordo com EMBRAPA (2009).
Obteve-se a condutividade elétrica (CE) em solução de substrato/água destilada de1:5 (v/v); o pH foi determinado em água, diluição com substrato/água destilada de 1:5 (v/v). A análise de teor total de sais solúveis (TTSS) determinado em água, diluição 1:10 (v/v) foi realizada no Laboratório de Substratos do Departamento de Horticultura e Silvicultura da Universidade Federal do Rio Grande do Sul (UFRGS) seguindose a metodologia proposta na Instrução Normativa SDA $\mathrm{N}^{\circ}$ 17 do Ministério da Agricultura, Pecuária e Abastecimento (MAPA, 2007).

Os dados provenientes da caracterização química dos 26 tratamentos com três repetições de três unidades amostrais para cada característica avaliada foram submetidos à análise de variância (ANOVA) $(\mathrm{p}<0,05)$ prosseguindo com o teste de Scott-Knott $(\mathrm{p}<0,05)$ para comparação de médias; além disto, a análise de Correlação de Pearson (r) foi realizada utilizando-se o software R (R Core Team, 2012).

\section{Resultados e Discussão}

As particularidades de cada componete do substrato podem fazer com que o $\mathrm{pH}$ seja elevado ou baixo, justificando a mistura entre os componentes para a obtenção de um pH adequado. No presente estudo os resíduos orgânicos e a vermiculita foram responsáveis pelo aumento do $\mathrm{pH}$ dos substratos; contrariamente, o aumento das proporções de lodo de esgoto favoreceu a diminuição do pH (Tabela 2).

Tabela 2. Média e desvio padrão das características químicas disponíveis $(\mathrm{P}, \mathrm{KeCa})$ dos substratos formulados com lodo de esgoto

\begin{tabular}{|c|c|c|c|c|}
\hline Tratamentos & $\begin{array}{c}\mathrm{pH} \\
\left(\mathrm{H}_{2} \mathrm{O}\right)\end{array}$ & $\left(\mathrm{mg} \mathrm{dm}^{-3}\right)$ & K & $\begin{array}{c}\mathrm{Ca} \\
\left(\mathrm{cmol}_{\mathrm{c}} \mathrm{dm}^{-3}\right)\end{array}$ \\
\hline T2-80\% LE /20\% FC & $4,6 m \pm 0,02$ & $159 \mathrm{f} \pm 1,60$ & $242 \mathrm{~g} \pm 9,24$ & $6,2 c \pm 0,50$ \\
\hline T4-40\% LE /60\% FC & $4,8 \mathrm{k} \pm 0,01$ & $144 \mathrm{~g} \pm 2,33$ & $967 \mathrm{e} \pm 90,17$ & $5,2 d \pm 0,46$ \\
\hline T5-20\% LE /80\% FC & $5,0 \mathrm{i} \pm 0,01$ & $135 \mathrm{~g} \pm 4,33$ & 968 e $\pm 149,69$ & $4,4 e \pm 0,11$ \\
\hline T6-80\% LE /20\% VER & $4,8 \mathrm{k} \pm 0,04$ & $145 \mathrm{~g} \pm 1,63$ & $47 \mathrm{~g} \pm 4,36$ & $7,7 b \pm 0,54$ \\
\hline T7-60\% LE /40\% VER & $4,9 \mathrm{j} \pm 0,01$ & $139 \mathrm{~g} \pm 0,52$ & $40 \mathrm{~g} \pm 1,53$ & $6,4 c \pm 0,54$ \\
\hline T9-20\% LE /80\% VER & $5,6 f \pm 0,01$ & $85 I \pm 19,37$ & $28 \mathrm{~g} \pm 5,77$ & $3,1 \mathrm{f} \pm 0,50$ \\
\hline T10-80\% LE /20\% PCN & $5,3 \mathrm{~g} \pm 0,01$ & $172 f \pm 2,40$ & $768 \mathrm{e} \pm 66,01$ & $6,0 \mathrm{c} \pm 2,25$ \\
\hline T11-60\% LE /40\% PCN & $6,2 d \pm 0,02$ & $161 \mathrm{f} \pm 10,75$ & $1586 c \pm 132,51$ & $5,8 d \pm 0,28$ \\
\hline T12-40\% LE /60\% PCN & $7,0 b \pm 0,03$ & $177 f \pm 5,58$ & $2613 b \pm 345,41$ & $4,4 \mathrm{e} \pm 0,20$ \\
\hline T13-20\% LE /80\% PCN & $7,3 a \pm 0,01$ & $228 d \pm 24,68$ & $8273 a \pm 649,33$ & $3,3 f \pm 0,46$ \\
\hline T14-80\% LE /20\% CO & $5,0 \mathrm{i} \pm 0,06$ & $172 f \pm 3,06$ & $492 f \pm 33,05$ & $6,2 c \pm 0,55$ \\
\hline T15-60\% LE / $40 \%$ CO & $5,2 \mathrm{~h} \pm 0,05$ & 206 e $\pm 3,11$ & 1031 e $\pm 55,75$ & $7,2 \mathrm{c} \pm 0,61$ \\
\hline $\mathrm{T} 20-40 \%$ LE $/ 60 \%$ CAC & $5,1 \mathrm{~h} \pm 0,04$ & $201 e \pm 20,79$ & $600 f \pm 37,50$ & $8,3 b \pm 0,80$ \\
\hline $\mathrm{T} 21-20 \%$ LE $/ 80 \%$ CAC & $5,7 e \pm 0,08$ & $243 d \pm 24,88$ & 878 e $\pm 73,66$ & $5,6 d \pm 0,45$ \\
\hline T22-80\% LE /20\% CAN & $4,5 \mathrm{~m} \pm, 09$ & $160 f \pm 3,32$ & $202 \mathrm{~g} \pm 22,81$ & $8,5 b \pm 0,51$ \\
\hline T23-60\% LE /40\% CAN & $4,9 j \pm 0,04$ & $165 f \pm 8,61$ & $360 \mathrm{~g} \pm 29,02$ & $6,8 c \pm 0,89$ \\
\hline $\mathrm{T} 24-40 \%$ LE $/ 60 \%$ CAN & $5,4 \mathrm{~g} \pm 0,06$ & $176 \mathrm{f} \pm 12,73$ & $499 f \pm 36,86$ & $4,1 \mathrm{e} \pm 0,99$ \\
\hline T25-20\% LE /80\% CAN & $6,2 d \pm 0,04$ & $245 d \pm 13,50$ & $1376 d \pm 74,89$ & $2,7 f \pm 0,23$ \\
\hline T26-100\% SC & $5,2 \mathrm{~h} \pm 0,03$ & $509 a \pm 17,69$ & $1325 d \pm 102,59$ & $12 \mathrm{a} \pm 1,11$ \\
\hline $\mathrm{CV}(\%)^{\star \star}$ & 13,33 & 43,60 & 156,68 & 32,86 \\
\hline
\end{tabular}

LE - Lodo de esgoto; FC - Fibra de coco triturada; VER - Vermiculita; PCN - Palha de café in natura; CO - Composto orgânico; CAC - Casca de arroz carbonizada; CAN - Casca de arroz in natura; SC - Substrato comercial; CTC - Capacidade de troca catiônica; CE - Condutividade elétrica; TTSS - Teor totais de sais solúveis

*Médias seguidas da mesma letra, na coluna, não diferem estatisticamente entre si pelo teste Scott-Knott $(p<0,05)$

**Coeficiente de variação 
Kämpf (2005) considera que o $\mathrm{pH}$ adequado do substrato deve estar entre 5 a 5,8 . Neste contexto, os substratos formulados com o composto orgânico se destacam nesta faixa de $\mathrm{pH}$ adequado conforme verificado na Tabela 2. Maiores proporções de resíduos na formulação dos substratos proporcionaram $\mathrm{pH}$ adequado, com exceção da palha de café in natura, em que as menores proporções foram as que beneficiaram o valor ideal de $\mathrm{pH}$ dos substratos. $\mathrm{O} \mathrm{pH}$ dos substratos obteve correlação significativa e negativa com os teores de Ca (Tabela 3). É possível verificar que o $\mathrm{pH}$ baixo está ligado a baixos teores de Ca e que altos teores de Ca resultam em $\mathrm{pH}$ mais elevado.

Corroborando com o presente estudo em que as maiores proporções de CAC elevaram o $\mathrm{pH}$ dos substratos, Kratz et al. (2012) utilizaram diferentes granulometrias de casca de arroz carbonizada, pura ou em mistura com fibra de coco, substrato comercial como componentes de substratos para produção de mudas de Eucalyptus benthamii x E. dunnii e constataram que a casca de arroz carbonizada apresentou $\mathrm{pH}$ mais elevado $(8,58)$ enquanto para a fibra de coco $(5,81)$ e os substratos comerciais à base de casca de pinus foram verificados menores valores de 5,30 e 6,06, fato que pode estar relacionado com o manejo da carbonização da casca de arroz adotado; a variação entre os substratos comerciais pode estar relacionada com a metodologia empregada na indústria.

De acordo com a classificação de Barros \& Novais (1999) os níveis críticos de $\mathrm{P}$ para utilização como substrato para produção de mudas de eucalipto, devem ser de $>0,19 \mathrm{cmol}_{c} \mathrm{dm}^{-3}$, para solos argilosos e de $>0,26 \mathrm{cmol}_{c} \mathrm{dm}^{-3}$, para solos arenosos; neste sentido, todos os tratamentos formulados estão acima do nível crítico para o $P$ disponível de vez que os valores variaram entre 0,27 e 1,64 $\mathrm{cmol}_{c} \mathrm{dm}^{-3}$ (Tabela 2).

Constatou-se correlação positiva e significativa entre os teores de fósforo e os teores de $\mathrm{Ca}(\mathrm{r}=0,49$, Tabela 3$)$ entre os substratos e proporções, embora para os tratamentos com fibra de coco, vermiculita, palha de café e casca de arroz in natura não tenha observada diferença significativa entre as proporções de 20, 40 e $60 \%$ de cada tratamento (Tabela 2).

$\mathrm{O}$ teor de fósforo nos substratos formulados a partir de vermiculita e fibra de coco diminuiu em função do aumento desses resíduos, possivelmente por apresentarem as menores médias para o fósforo disponível. Deste modo, os altos teores de $P$ nesses substratos podem ser atribuídos ao lodo de esgoto. Tal resíduo é reputado por apresentar matéria orgânica prontamente mineralizável e altos teores de nutrientes, como o fósforo (Corrêa et al., 2005; 2010; Araújo et al., 2009).
De acordo com a classificação de Gonçalves \& Poggiani (1996) para a produção de mudas florestais o teor de potássio disponível do substrato deve situar-se entre 3 a $10 \mathrm{cmol}_{c} \mathrm{dm}^{-3}$. A maioria dos tratamentos possui baixa concentração de potássio e apenas os substratos formulados com palha de café in natura nas proporções de 40 e 60\%, composto orgânico (60 e 80\%), casca de arroz não carbonizada (80\%) e substrato comercial (100\%) apresentaram níveis adequados de potássio (Tabela 2). Estudos confirmam que o lodo de esgoto não é uma boa fonte de potássio devido à sua baixa concentração no mesmo (Vieira et al., 2004; Silva et al., 2008; Paiva et al., 2009; Oro et al., 2012).

A palha de café in natura é fonte de potássio para os substratos formulados com este resíduo e as proporções de 60 e $80 \%$ foram superiores às dos demais tratamentos, com médias de 6,68 e $21,16 \mathrm{cmol}_{\mathrm{c}} \mathrm{dm}^{-3}$, respectivamente. O potássio se correlacionou significativa e positivamente, com o pH dos substratos $(\mathrm{r}=$ 0,77). Mesmo havendo correlação positiva entre o pH e o K, sua absorção pelas plantas pode estar ligada a fatores outros, como teores tóxicos de $\mathrm{Al}$ que inibem seu crescimento pela destruição do meristema apical das raízes, o aumento do $\mathrm{pH}$ e resultante aumento do K podem diminuir os teores tóxicos de $\mathrm{Al}$ (Tabela 3).

Com o objetivo de avaliar o desenvolvimento de orquídea híbrida (C. forbesiix C. labiata) x C. labiata em substratos à base de xaxim desfibrado; palha de café; palha de café + coco em pó; palha de café + fibra de coco; palha de café + casca de pinus; palha de café + casca de arroz carbonizada, Assis et al. (2011) afirmam que a palha de café possui teores de potássio mais elevados que os demais substratos, conforme também foi possível observar no presente estudo.

No que se refere aos teores disponíveis de $\mathrm{Ca}^{+2}$, Gonçalves \& Poggiani (1996) sugerem que o teor de Ca disponível no substrato está entre 10 e $20 \mathrm{cmol}_{\mathrm{c}} \mathrm{dm}^{-3}$. Apenas no substrato comercial ( $\mathrm{T}$ 26) o teor de cálcio é considerado adequado enquanto os demais podem ser considerados baixos; mesmo assim, a adição de lodo de esgoto contribuiu para o aumento do teor de $\mathrm{Ca}^{+2}$ nos substratos.

O nível considerado adequado para o teor de $\mathrm{Mg}^{+2}$ disponível de acordo com Barros \& Novais (1999) deve situar-se em um limite superior a $0,05 \mathrm{cmol}_{c} \mathrm{dm}^{3}$ sentido em que todos os substratos formulados estão acima do nível crítico considerado pelos autores supracitados. A utilização dos resíduos fibra de coco, composto orgânico, casca de arroz carbonizada e a vermiculita foram benéficos para o fornecimento de $\mathrm{Mg}^{+2}$ para os substratos, à medida que suas maiores proporções foram adicionadas (Tabela 4 ).

Tabela 3. Correlação entre o pH e os teores disponíveis dos substratos $\mathrm{P}, \mathrm{K}, \mathrm{Ca}, \mathrm{Mg}$, condutividade elétrica (CE) e teor total de sais solúveis (TTSS) nos diferentes substratos

\begin{tabular}{|c|c|c|c|c|c|c|c|}
\hline & $\mathrm{pH}$ & $\mathbf{P}$ & $\bar{K}$ & $\mathrm{Ca}$ & $\mathrm{Mg}$ & $\overline{C E}$ & TTSS \\
\hline $\mathrm{pH}$ & $1,00^{\star \star}$ & & & & & & \\
\hline K & $0,77^{\star \star}$ & $0,28^{\text {ns }}$ & 1,00 ** & & & & \\
\hline $\mathrm{Mg}$ & $0,16^{\mathrm{ns}}$ & $0,57^{\mathrm{ns}}$ & $0,07^{\mathrm{ns}}$ & $0,30^{\text {ns }}$ & $1,00^{* *}$ & & \\
\hline CE & $-0,27^{\mathrm{ns}}$ & 0,51 ** & $-0,00^{\mathrm{ns}}$ & $0,62^{\star \star}$ & $0,33^{\text {ns }}$ & $1,00 * *$ & \\
\hline TTSS & $-0,39 *$ & $0,43^{\star}$ & $-0,19^{\text {ns }}$ & $0,67^{* *}$ & $0,32^{\mathrm{ns}}$ & $0,76^{\star *}$ & 1,00 ** \\
\hline
\end{tabular}

** $e^{*}$ Significativo a nível de 0,01 e 0,05 de probabilidade; ns Não significativo $(p>0,05)$ 
Tabela 4. Média e desvio padrão das características químicas (Mg, CTC e CE) dos substratos formulados com lodo de esgoto

\begin{tabular}{|c|c|c|c|c|}
\hline \multirow{2}{*}{ Tratamentos } & $\mathrm{Mg}$ & CTC & \multirow{2}{*}{$\begin{array}{c}\text { CE } \\
\left(\mathrm{mS} \mathrm{cm}^{-1}\right)\end{array}$} & \multirow{2}{*}{$\begin{array}{c}\text { TTSS } \\
\left(\mathrm{g} \mathrm{L}^{-1}\right)\end{array}$} \\
\hline & \multicolumn{2}{|c|}{$\left(\mathrm{cmol}_{\mathrm{c}} \mathrm{dm}^{3}\right)$} & & \\
\hline T1-100\% LE & $1,0 * e \pm 0,17$ & $45,1 \mathrm{a} \pm 2,37$ & $0,28 j \pm 0,05$ & $1,99 a \pm 0,02$ \\
\hline T2-80\% LE /20\% FC & $1,0 \mathrm{e} \pm 0,14$ & $40,8 b \pm 2,68$ & $0,90 \mathrm{c} \pm 0,01$ & $1,74 b \pm 0,01$ \\
\hline T3-60\% LE /40\% FC & $1,4 \mathrm{e} \pm 0,30$ & $39,7 b \pm 1,31$ & $0,84 c \pm 0,01$ & $1,28 d \pm 0,02$ \\
\hline T4-40\% LE /60\% FC & $1,6 \mathrm{e} \pm 0,32$ & $41,5 b \pm 0,29$ & $0,73 d \pm 0,01$ & $0,87 \mathrm{f} \pm 0,01$ \\
\hline T5-20\% LE /80\% FC & $1,8 d \pm 0,29$ & $44,0 \mathrm{a} \pm 3,14$ & $0,67 \mathrm{e} \pm 0,01$ & $0,86 f \pm 0,48$ \\
\hline T6-80\% LE /20\% VER & $2,5 d \pm 0,41$ & $39,7 b \pm 1,07$ & $0,77 d \pm 0,03$ & $1,57 c \pm 0,15$ \\
\hline T7-60\% LE / $40 \%$ VER & $3,8 c \pm 0,25$ & $38,1 b \pm 1,74$ & $0,46 h \pm 0,02$ & $1,04 \mathrm{e} \pm 0,06$ \\
\hline T8-40\% LE /60\% VER & $4,5 c \pm 0,68$ & $35,1 b \pm 1,89$ & $0,26 j \pm 0,01$ & $0,37 \mathrm{~h} \pm 0,01$ \\
\hline T9-20\% LE /80\% VER & $5,1 b \pm 0,87$ & $29,8 c \pm 0,80$ & $0,20 k \pm 0,01$ & $0,19 \mathrm{i} \pm 0,00$ \\
\hline T10-80\% LE /20\% PCN & $1,6 \mathrm{e} \pm 0,48$ & $35,2 b \pm 9,77$ & $0,51 \mathrm{~g} \pm 0,00$ & $1,10 \mathrm{e} \pm 0,03$ \\
\hline T11-60\% LE /40\% PCN & $1,2 \mathrm{e} \pm 0,25$ & $41,9 b \pm 0,84$ & $0,43 h \pm 0,02$ & $0,77 \mathrm{~g} \pm 0,04$ \\
\hline $\mathrm{T} 12-40 \%$ LE $/ 60 \%$ PCN & $1,2 \mathrm{e} \pm 0,23$ & $38,0 b \pm 2,56$ & $0,42 h \pm 0,01$ & $0,63 \mathrm{~g} \pm 0,10$ \\
\hline T13-20\% LE /80\% PCN & $2,5 d \pm 0,40$ & $51,4 \mathrm{a} \pm 1,84$ & $0,51 \mathrm{~g} \pm 0,01$ & $0,42 h \pm 0,06$ \\
\hline T14-80\% LE /20\% CO & $2,4 d \pm 0,42$ & $41,8 b \pm 0,65$ & $0,79 d \pm 0,01$ & $1,72 b \pm 0,05$ \\
\hline T15-60\% LE /40\% CO & $5,7 b \pm 0,58$ & $39,3 b \pm 13,03$ & $0,87 c \pm 0,00$ & $1,80 b \pm 0,04$ \\
\hline T16-40\% LE /60\% C0 & $4,2 \mathrm{c} \pm 1,06$ & $26,1 \mathrm{c} \pm 2,19$ & $0,98 b \pm 0,01$ & $2,05 a \pm 0,06$ \\
\hline T17-20\% LE /80\% CO & $7,7 \mathrm{a} \pm 0,30$ & $26,6 \mathrm{c} \pm 2,35$ & $0,93 b \pm 0,01$ & $1,88 b \pm 0,02$ \\
\hline $\mathrm{T} 18-80 \%$ LE $/ 20 \%$ CAC & $1,4 \mathrm{e} \pm 0,21$ & $29,8 c \pm 0,45$ & $0,87 c \pm 0,12$ & $1,32 d \pm 0,02$ \\
\hline T19-60\% LE /40\% CAC & $1,4 \mathrm{e} \pm 0,25$ & $33,5 c \pm 0,88$ & $0,59 f \pm 0,01$ & $0,91 f \pm 0,04$ \\
\hline T20-40\% LE $/ 60 \%$ CAC & $1,8 d \pm 0,25$ & $34,7 b \pm 0,89$ & $0,44 h \pm 0,01$ & $0,52 h \pm 0,02$ \\
\hline $\mathrm{T} 21-20 \%$ LE $/ 80 \%$ CAC & $2,0 d \pm 0,47$ & $31,2 \mathrm{c} \pm 0,93$ & $0,33 \mathrm{i} \pm 0,01$ & $0,33 \mathrm{i} \pm 0,01$ \\
\hline T22-80\% LE /20\% CAN & $1,2 \mathrm{e} \pm 0,17$ & $32,2 \mathrm{c} \pm 0,85$ & $0,67 \mathrm{e} \pm 0,03$ & $1,28 d \pm 0,04$ \\
\hline T23-60\% LE /40\% CAN & $1,0 \mathrm{e} \pm 0,29$ & $26,0 \mathrm{c} \pm 0,75$ & 0,67 e $\pm 0,02$ & $1,31 d \pm 0,02$ \\
\hline T24-40\% LE /60\% CAN & $0,8 \mathrm{e} \pm 0,36$ & $20,6 d \pm 1,46$ & $0,58 f \pm 0,01$ & $0,77 \mathrm{~g} \pm 0,08$ \\
\hline T25-20\% LE $/ 80 \%$ CAN & $1,0 \mathrm{e} \pm 0,14$ & 14,0 e $\pm 0,82$ & $0,35 i \pm 0,02$ & $0,29 \mathrm{i} \pm 0,03$ \\
\hline $\mathrm{T} 26-100 \% \mathrm{SC}$ & $7,3 a \pm 0,63$ & $46,3 \mathrm{a} \pm 4,95$ & $1,14 a \pm 0,03$ & $2,14 a \pm 0,05$ \\
\hline $\operatorname{CV}(\%)^{\star \star}$ & 76,02 & 24,94 & 39,65 & 53,45 \\
\hline
\end{tabular}

CTC - Capacidade de troca catiônica; CE - Condutividade elétrica; TTSS - Teores totais de sais solúveis LE - Lodo de esgoto; FC - Fibra de coco triturada; VER - Vermiculita; PCN - Palha de café in natura; CO - Composto orgânico; CAC - Casca de arroz carbonizada; CAN - Casca de arroz in natura; SC - Substrato comercial

*Médias seguidas da mesma letra, na coluna, não diferem estatisticamente entre si pelo teste Scott-Knott $(p<0,05)$

${ }^{\star *}$ Coeficiente de variação

A CTC de todos os tratamentos, com exceção do tratamento T25 (20\% LE e 80\% CAN), pode ser considerada adequada ( $>20$ $\mathrm{cmol}_{\mathrm{c}} \mathrm{dm}^{-3}$ ) segundo critérios de Gonçalves \& Poggiani (1996). Os altos teores de CTC encontrados no presente estudo podem estar associados à matéria orgânica acrescida nos substratos pela adição de lodo de esgoto, como observado nos tratamentos formulados com vermiculita, composto orgânico e casca de arroz in natura. Os tratamentos T1 (100\% LE), T5 (20\% LE /80\% FC), T13 (20\% LE /80\% PCN) e T26 (100\% SC) apresentaram as maiores médias para esta característica (Tabela 4).

A utilização de resíduos orgânicos, em particular o lodo de esgoto possuem, por conterem altas concentrações de matéria orgânica, grandes superfícies de troca responsáveis pelo aumento da CTC; neste estudo a incorporação de lodo de esgoto nos substratos com vermiculita aumentou sua CTC; assim, quando se elevam as proporções de lodo de esgoto e se diminui a de vermiculita, ocorre decréscimo de CTC do substrato, fato que também é observado com a utilização dos resíduos de composto orgânico e casca de arroz in natura.

A condutividade elétrica do substrato não deve exceder o limite de $1,0 \mathrm{mS} \mathrm{cm}^{-1}$ segundo Gonçalves et al. (2000). O maior valor obtido foi apenas para o substrato comercial. Segundo Kämpf (2005) altos valores de salinidade podem danificar as raízes impedindo a absorção de água e nutriente, razão por que tal evento é um dos itens que devem ser considerados na escolha do material. Com exceção do tratamento T13 (20\% LE e 80\% PCN) e dos tratamentos formulados com composto orgânico, maiores proporções do lodo de esgoto resultaram em menores médias para a CE; entretanto, o lodo de esgoto foi responsável pelos maiores teores de condutividade elétrica nos demais substratos em que suas maiores proporções resultaram em maiores médias para a CE (Tabela 4). Backes et al. (2009) relatam que a adição de lodo de esgoto e a maior liberação de nutrientes aumentaram a condutividade elétrica do solo. A condutividade elétrica obteve correlação positiva e significativa com os teores disponíveis de $\mathrm{P}(\mathrm{r}$ $=0,51) \mathrm{e} \mathrm{Ca}(\mathrm{r}=0,62)$ pelo fato, possivelmente, da condutividade elétrica estar ligada ao teor de sais do extrato (Tabela 3).

A salinidade dos substratos formulados com $100 \%$ de substrato comercial e $60 \%$ de composto orgânico pode ser classificada alta (2,0 a 4,0 $\mathrm{g} \mathrm{L}^{-1}$, segundo Kämpf (2005) de vez que foram registrados valores de 2,14 e 2,05 $\mathrm{g} \mathrm{L}^{-1}$, respectivamente. Os substratos formulados sobretudo com maiores proporções de lodo de esgoto apresentam salinidade média ou normal. Por outro lado, aqueles formulados com menores proporções de LE, possivelmente por apresentarem menos teores de $\mathrm{Ca}^{2+}$ e $\mathrm{P}$ disponíveis, possuem salinidade abaixo de $1 \mathrm{~g} \mathrm{~L}^{-1}$, valor considerado baixo de acordo com Kämpf (2005).

A salinidade dos substratos está correlacionada positivamente com os teores de $\mathrm{P}(\mathrm{r}=0,43)$, $\mathrm{Ca}(\mathrm{r}=0,67)$ e $\mathrm{CE}(\mathrm{r}=0,76)$ e negativamente com o pH $(r=-0,39)$ (Tabela 3). O aumento e a diminuição dos teores de $\mathrm{P}$ e $\mathrm{Ca}^{2+}$ estão correlacionados à condutividade elétrica dos substratos e esta, por sua vez, serve de base para o cálculo da salinidade dos substratos (Kämpf, 2005) o que pode explicar a correlação positiva entre os atributos avaliados. 
Os substratos formulados apresentaram diferenças significativas entre os teores totais de $\mathrm{N}$, com as maiores médias entre 19 e $21,1 \mathrm{~g} \mathrm{~kg}^{-1}$ para os substratos com $100 \%$ de lodo de esgoto (T1) enquanto aqueles formulados com palha de café in natura nas proporções 20,40 e $60 \%$, não diferindo estatisticamente entre si ( $\mathrm{p} \leq 0,05$, Tabela 5).

Higashikawa et al. (2010) caracterizaram as propriedades químicas de resíduos orgânicos e encontraram valores totais de $\mathrm{N}$ na casca de café, esterco bovino e lodo de esgoto acima de 18 e de até $12,2 \mathrm{~g} \mathrm{~kg}^{-1} \mathrm{em}$ fibra de coco, casca de pinho, serragem, turfa, carvão e vermiculita enquanto no presente estudo o lodo de esgoto apresentou uma média de $19 \mathrm{~g} \mathrm{~kg}^{-1}$ e para a fibra de coco este valor se manteve entre 11,2 a $16,4 \mathrm{~g} \mathrm{~kg}^{-1}$, em que o maior valor de $\mathrm{N}$ foi obtido nos substratos com maiores proporções de lodo de esgoto.
A concentração de $\mathrm{P}$ total nos substratos variou pouco entre as proporções dos resíduos nos substratos (Tabela 5). A concentração de $\mathrm{P}$ total dos substratos apresentou correlação positiva com teores totais de $\mathrm{N}(\mathrm{r}=0,78)$ e de forma negativa com o $\mathrm{K}(\mathrm{r}=-0,41)$ (Tabela 6). Os substratos formulados com diferentes proporções de vermiculita não diferiram entre si e os formulados com fibra de coco, composto orgânico e casca de arroz in natura, tiveram o teor total de $\mathrm{P}$ diminuído com o acréscimo dos mesmos. É provável que esta relação ocorre em função dos baixos valores de $\mathrm{P}$ disponíveis nesses resíduos. Higashikawa et al. (2010) encontraram o valor total de P de $4 \mathrm{~g}$ $\mathrm{kg}^{-1}$, enquanto para o lodo de esgoto no presente estudo o valor foi de $4,9 \mathrm{~g} \mathrm{~kg}^{-1}$ para este resíduo.

Os tratamentos formulados com fibra de coco, palha de café in natura, casca de arroz carbonizada e in natura, elevaram

Tabela 5. Médias e desvio padrão dos teores totais de macronutrientes dos substratos formulados com lodo de esgoto

\begin{tabular}{|c|c|c|c|c|c|c|}
\hline \multirow{2}{*}{ Tratamentos } & $\mathbf{N}$ & $\mathbf{P}$ & K & $\mathrm{Ca}$ & $\mathrm{Mg}$ & $S$ \\
\hline & \multicolumn{6}{|c|}{$\left(\mathrm{g} \mathrm{kg}^{-1}\right)$} \\
\hline T1-100\% LE & $19,0 \mathrm{a} \pm 0,07$ & $04,9 a \pm 0,03$ & $01,1 \mathrm{e} \pm 0,03$ & $03,9 c \pm 0,06$ & $02,1 \mathrm{c} \pm 0,02$ & $05,4 a \pm 0,08$ \\
\hline T2-80\% LE /20\% FC & $16,4 b \pm 0,28$ & $04,2 \mathrm{a} \pm 0,01$ & 01,3 e $\pm 0,02$ & $03,8 c \pm 0,05$ & $01,9 \mathrm{c} \pm 0,02$ & $05,4 a \pm 0,14$ \\
\hline T3-60\% LE $/ 40 \%$ FC & $14,0 \mathrm{c} \pm 0,13$ & $03,9 a \pm 0,05$ & $01,6 d \pm 0,04$ & $05,0 \mathrm{c} \pm 0,07$ & $01,9 c \pm 0,02$ & $04,4 a \pm 0,09$ \\
\hline T4-40\% LE /60\% FC & $13,2 c \pm 0,10$ & $03,9 a \pm 0,06$ & $02,6 d \pm 0,05$ & $05,0 \mathrm{c} \pm 0,05$ & $01,8 c \pm 0,06$ & $04,3 \mathrm{a} \pm 0,09$ \\
\hline T5-20\% LE /80\% FC & $11,2 c \pm 0,03$ & $03,3 b \pm 0,02$ & $04,5 b \pm 0,04$ & $04,7 c \pm 0,10$ & $02,2 \mathrm{c} \pm 0,02$ & $04,2 \mathrm{a} \pm 0,09$ \\
\hline T6-80\% LE /20\% VER & $16,3 b \pm 0,18$ & $04,4 a \pm 0,04$ & $0,9 \mathrm{e} \pm 0,02$ & $03,8 c \pm 0,06$ & $04,5 \mathrm{c} \pm 0,06$ & $05,7 a \pm 0,28$ \\
\hline T7-60\% LE / $40 \%$ VER & $16,5 b \pm 0,00$ & $04,2 \mathrm{a} \pm 0,03$ & 01,0 e $\pm 0,03$ & $03,7 c \pm 0,04$ & $09,0 b \pm 0,25$ & $05,3 a \pm 0,24$ \\
\hline T8-40\% LE /60\% VER & $15,9 b \pm 0,09$ & $03,9 a \pm 0,05$ & $0,8 \mathrm{e} \pm 0,02$ & $04,6 c \pm 0,11$ & $11,6 b \pm 1,05$ & $04,6 a \pm 0,07$ \\
\hline T9-20\% LE /80\% VER & $13,7 c \pm 0,07$ & $04,5 a \pm 0,33$ & $0,6 \mathrm{e} \pm 0,01$ & $02,8 c \pm 0,03$ & $19,9 a \pm 2,33$ & $03,7 \mathrm{a} \pm 0,12$ \\
\hline $\mathrm{T} 10-80 \%$ LE $/ 20 \%$ PCN & $20,7 a \pm 0,07$ & $05,3 a \pm 0,01$ & $02,2 d \pm 0,03$ & $04,7 \mathrm{c} \pm 0,15$ & $02,5 \mathrm{c} \pm 0,00$ & $04,6 a \pm 0,04$ \\
\hline T11-60\% LE /40\% PCN & $20,1 \mathrm{a} \pm 0,02$ & $04,8 \mathrm{a} \pm 0,07$ & $03,6 c \pm 0,05$ & $03,6 c \pm 0,03$ & $02,2 \mathrm{c} \pm 0,02$ & $05,2 \mathrm{a} \pm 0,11$ \\
\hline T12-40\% LE /60\% PCN & $21,1 \mathrm{a} \pm 0,12$ & $04,9 a \pm 0,02$ & $04,7 b \pm 0,03$ & $03,8 c \pm 0,03$ & $02,2 \mathrm{c} \pm 0,01$ & $05,0 a \pm 0,08$ \\
\hline T13-20\% LE /80\% PCN & $17,7 b \pm 0,12$ & $02,7 b \pm 0,01$ & $07,4 a \pm 0,28$ & $03,8 \mathrm{c} \pm 0,02$ & $01,6 \mathrm{c} \pm 0,00$ & $03,8 a \pm 0,15$ \\
\hline T14-80\% LE /20\% C0 & $17,6 b \pm 0,14$ & $04,3 \mathrm{a} \pm 0,04$ & $02,0 d \pm 0,04$ & $04,3 c \pm 0,11$ & $02,7 \mathrm{c} \pm 0,03$ & $04,6 \mathrm{a} \pm 0,11$ \\
\hline T15-60\% LE $/ 40 \%$ CO & $15,4 b \pm 0,42$ & $03,5 b \pm 0,07$ & $02,9 \mathrm{c} \pm 0,05$ & $06,5 \mathrm{c} \pm 0,13$ & $03,5 c \pm 0,03$ & $03,2 \mathrm{a} \pm 0,12$ \\
\hline T16-40\% LE /60\% C0 & $09,2 d \pm 0,44$ & $02,6 b \pm 0,02$ & $03,6 c \pm 0,08$ & $10,1 b \pm 0,07$ & $04,5 \mathrm{c} \pm 0,02$ & $02,0 \mathrm{a} \pm 0,03$ \\
\hline T17-20\% LE $/ 80 \%$ CO & $09,3 d \pm 0,25$ & $02,1 b \pm 0,01$ & $04,4 b \pm 0,01$ & $07,8 b \pm 0,22$ & $04,6 \mathrm{c} \pm 0,02$ & $01,7 \mathrm{a} \pm 0,04$ \\
\hline T18-80\% LE /20\% CAC & $14,5 \mathrm{c} \pm 0,41$ & $03,7 \mathrm{a} \pm 0,01$ & 01,0 e $\pm 0,02$ & $12,7 \mathrm{a} \pm 0,36$ & $02,7 \mathrm{c} \pm 0,02$ & $03,2 \mathrm{a} \pm 0,03$ \\
\hline $\mathrm{T} 19-60 \%$ LE $/ 40 \%$ CAC & $16,8 b \pm 0,58$ & $03,8 a \pm 0,08$ & $01,2 \mathrm{e} \pm 0,03$ & $09,2 b \pm 0,17$ & $02,6 \mathrm{c} \pm 0,02$ & $03,3 a \pm 0,02$ \\
\hline T20-40\% LE $/ 60 \%$ CAC & $13,9 c \pm 0,18$ & $03,3 b \pm 0,05$ & 01,3 e $\pm 0,01$ & $07,8 b \pm 0,08$ & $02,2 c \pm 0,02$ & $02,8 \mathrm{a} \pm 0,02$ \\
\hline T21-20\% LE $/ 80 \%$ CAC & $12,7 c \pm 0,02$ & $03,2 b \pm 0,00$ & $01,9 d \pm 0,02$ & $05,5 \mathrm{c} \pm 0,07$ & $02,1 \mathrm{c} \pm 0,01$ & $03,0 \mathrm{a} \pm 0,05$ \\
\hline T22-80\% LE /20\% CAN & $14,2 \mathrm{c} \pm 0,19$ & $03,9 a \pm 0,03$ & 01,3 e $\pm 0,01$ & $12,7 \mathrm{a} \pm 0,42$ & $02,8 \mathrm{c} \pm 0,02$ & $04,5 a \pm 0,12$ \\
\hline T23-60\% LE /40\% CAN & $13,1 c \pm 0,21$ & $03,2 b \pm 0,06$ & 01,5 e $\pm 0,02$ & $11,9 a \pm 0,45$ & $03,0 \mathrm{c} \pm 0,05$ & $04,5 a \pm 0,14$ \\
\hline T24-40\% LE /60\% CAN & $11,3 c \pm 0,42$ & $02,8 b \pm 0,03$ & $02,0 d \pm 0,02$ & $09,2 b \pm 0,22$ & $02,2 c \pm 0,02$ & $05,8 \mathrm{a} \pm 0,37$ \\
\hline T25-20\% LE /80\% CAN & $08,8 d \pm 0,19$ & $02,4 b \pm 0,01$ & $04,1 b \pm 0,02$ & $04,8 \mathrm{c} \pm 0,07$ & $02,0 \mathrm{c} \pm 0,01$ & $04,6 a \pm 0,30$ \\
\hline T26-100\% SC & $06,6 d \pm 0,23$ & $03,4 b \pm 0,03$ & $03,3 c \pm 0,02$ & $10,7 a \pm 0,16$ & $11,6 b \pm 0,23$ & $06,4 \mathrm{a} \pm 0,31$ \\
\hline $\operatorname{CV}(\%)^{\star \star}$ & 28,77 & 27,46 & 69,07 & 52,91 & 139,34 & 39,87 \\
\hline
\end{tabular}

LE - lodo de esgoto; FC - fibra de coco triturada; VER - vermiculita; PCN - palha de café in natura; CO - composto orgânico; CAC - casca de arroz carbonizada; CAN - casca de arroz in natura; SC - substrato comercial; *Médias seguidas da mesma letra, na coluna não diferem estatisticamente entre si pelo teste Scott-Knott $(p<0,05)$; ${ }^{* *}$ Coeficiente de variação

Tabela 6. Correlação entre teores totais de macro e micronutrientes

\begin{tabular}{|c|c|c|c|c|c|c|c|c|c|c|c|}
\hline & $\mathbf{N}$ & $\mathbf{P}$ & $\bar{K}$ & $\mathrm{Ca}$ & $\mathrm{Mg}$ & $S$ & $\mathrm{Zn}$ & $\mathrm{Fe}$ & $\mathrm{Mn}$ & $\mathrm{Cu}$ & B \\
\hline $\mathrm{N}$ & 1,00 ** & & & & & & & & & & \\
\hline $\mathrm{P}$ & $0,78^{\star *}$ & $1,00^{\star *}$ & & & & & & & & & \\
\hline K & $-0,11^{\mathrm{ns}}$ & $-0,41^{*}$ & $1,00 * *$ & & & & & & & & \\
\hline $\mathrm{Ca}$ & $-0,47^{\star}$ & $-0,42^{\star}$ & $-0,14^{\mathrm{ns}}$ & $1,00 * *$ & & & & & & & \\
\hline $\mathrm{Mg}$ & $-0,20^{\text {ns }}$ & $0,12^{\text {ns }}$ & $-0,28^{\text {ns }}$ & $-0,11^{\text {ns }}$ & 1,00 ** & & & & & & \\
\hline$S$ & $0,23^{\text {ns }}$ & 0,48 * & $-0,16^{\mathrm{ns}}$ & $-0,24^{\mathrm{ns}}$ & $0,07^{\mathrm{ns}}$ & $1,00^{* *}$ & & & & & \\
\hline $\mathrm{Zn}$ & $0,64^{* *}$ & 0,60 ** & $-0,58 * *$ & $0,15^{\mathrm{ns}}$ & $-0,30^{\text {ns }}$ & $0,15^{\text {ns }}$ & $1,00 * *$ & & & & \\
\hline $\mathrm{Fe}$ & $-0,31^{\mathrm{ns}}$ & $0,06^{\text {ns }}$ & $0,03^{\text {ns }}$ & $0,23^{\text {ns }}$ & $0,38^{*}$ & $0,42^{*}$ & $-0,32^{\mathrm{ns}}$ & $1,00^{\star *}$ & & & \\
\hline $\mathrm{Mn}$ & $-0,67 * \star$ & $-0,64$ ** & $0,14^{\mathrm{ns}}$ & $0,37^{\mathrm{ns}}$ & $-0,05$ & $-0,14^{\mathrm{ns}}$ & $-0,27^{\mathrm{ns}}$ & $0,11^{\text {ns }}$ & 1,00 ** & & \\
\hline $\mathrm{Cu}$ & 0,60 ** & $0,77^{* *}$ & $-0,25^{\mathrm{ns}}$ & $-0,45^{\star}$ & $-0,17$ & $0,43^{*}$ & $0,43^{*}$ & $-0,04^{\text {ns }}$ & $-0,61^{* *}$ & 1,00 ** & \\
\hline B & $-0,26^{\mathrm{ns}}$ & $-0,33^{\text {ns }}$ & $0,75^{*}$ & $-0,05^{\mathrm{ns}}$ & $-0,09$ & $-0,06^{\mathrm{ns}}$ & $-0,65^{\star *}$ & $0,31^{\text {ns }}$ & $-0,11^{\mathrm{ns}}$ & $-0,03^{\text {ns }}$ & 1,00 ** \\
\hline
\end{tabular}

Teste t: **Significativo a nível de $1 \%$ de probabilidade; *Significativo a nível de $5 \%$ de probabilidade; nsNão significativo $(p<0,05)$ 
o teor total de $\mathrm{K}$ sempre que eram adicionadas ao substrato. Corroborando com o presente estudo Higashikawa et al. (2010) também verificaram que a palha de café in natura teve a maior concentração de $\mathrm{K}$ de todos os resíduos indicando que este resíduo é bom fornecedor de $\mathrm{K}$.

Os tratamentos T18 (80\% LE /20\% CAC), T22 (80\% LE / $20 \%$ CAN), T23 (60\% LE /40\% CAN) e T26 (100\% SC) proporcionaram as maiores médias de teor total de $\mathrm{Ca}$ não havendo diferença significativa entre tais substratos ( $\mathrm{p} \leq 0,05$, Tabela 5 ). Tal fato pode estar relacionado com a capacidade de fornecimento de Ca pela casca de arroz e por meio de adidivos no substrato comercial. Os teores totais de $\mathrm{Ca}$ se correlacionaram negativamente com os teores de $\mathrm{N}(\mathrm{r}=-0,47)$ e com os teores totais de $\mathrm{P}(\mathrm{r}=-0,42)$ conforme se observa na Tabela 6 .

As maiores médias para os teores totais de $\mathrm{Mg}$ foram encontradas nos substratos formulados a partir de vermiculita, fato que pode ser explicado em virtude da sua constituição mineralógica pois, segundo Ugarte et al. (2005) a vermiculita é um silicato hidratado de magnésio, alumínio e ferro. O substrado com $80 \%$ de vermiculita propiciou a maior média de teores totais de $\mathrm{Mg}$ (19,9 $\left.\mathrm{g} \mathrm{kg}^{-1}\right)$ havendo diferença significativa em relação aos valores dos demais tratamentos. Apesar de ser uma fonte de magnésio, a vermiculita é um material não renovável, o que justifica a realização de experimentos utilizando-se resíduos orgânicos (Tabela 5).

$\mathrm{O}$ teor de Ca e de $\mathrm{Mg}$ do lodo de esgoto do presente estudo foi 3,9 e 2,1 $\mathrm{g} \mathrm{kg}^{-1}$ respectivamente, valor inferior para o teor de
Ca e superior para o teor de $\mathrm{Mg}$ ao encontrado por Higashikawa et al. (2010) que foi de 23,4 e 1,9 $\mathrm{g} \mathrm{kg}^{-1}$, respectivamente, e inferior ao observado por Guerrini \& Trigueiro (2004) os quais registraram médias de 16,7 para Ca e 2,2 $\mathrm{g} \mathrm{kg}^{-1}$ para $\mathrm{Mg}$, o que pode estar relacionado com o manejo de tratamento, processo de secagem e armazenamento, até sua utilização.

Os teores totais de $\mathrm{S}$ não diferiram estatisticamente entre si nas formulações dos substratos (Tabela 5). Mesmo não havendo diferenças significativas entre as médias, o substrato comercial obteve a maior média de $\mathrm{S}$ total em razão, possivelmente, do gesso agrícola e aditivos de sua composição. Os diferentes componentes dos substratos apresentam características próprias; deste modo se supunha que as diferentes proporções dos resíduos resultassem em diferenças significativas nas médias, o que não foi observado, contrariamente ao observado no referido estudo no qual não se constataram diferenças entre as médias mas Guerrini \& Trigueiro (2004) registraram teor de $10,1 \mathrm{~g} \mathrm{~kg}^{-1}$ para o lodo de esgoto e $0,2 \mathrm{~g} \mathrm{~kg}^{-1}$ para a casca de arroz carbonizada. Nos substratos compostos desses dois componentes nas proporções de $90,80,60$ e $50 \%$ de lodo de esgoto, os teores de $\mathrm{S}$ foram superiores aos do lodo de esgoto puro.

O lodo de esgoto e a palha de café in natura foram responsáveis por elevar os teores totais dos micronutrientes Zn e Fe nos substratos com maior proporção (80\%) de resíduos (Tabela 7). Guerrini \& Trigueiro (2004) encontraram resultados semelhantes aos do presente estudo em que o lodo de esgoto também proporcionou maior média para esses nutrientes,

Tabela 7. Médias e desvio padrão dos teores totais de Zn, Fe, Mn, Cu e B dos substratos formulados com lodo de esgoto

\begin{tabular}{|c|c|c|c|c|c|}
\hline \multirow{2}{*}{ Tratamentos } & $\mathrm{Zn}$ & $\mathrm{Fe}$ & Mn & $\mathrm{Cu}$ & B \\
\hline & \multicolumn{5}{|c|}{$\left(\mathrm{mg} \mathrm{kg}^{-1}\right)$} \\
\hline T1-100\% LE & $316 a \pm 37,67$ & $32000 \mathrm{a} \pm 1802,8$ & $137 d \pm 7,3$ & $116 a \pm 8,64$ & $14 c \pm 6,93$ \\
\hline T2-80\% LE /20\% FC & $266 b \pm 19,79$ & $29883 b \pm 1336,4$ & $127 d \pm 9,0$ & $75 a \pm 48,55$ & $9 c \pm 2,28$ \\
\hline T3-60\% LE /40\% FC & $239 b \pm 29,47$ & $25700 \mathrm{c} \pm 1969,8$ & $126 d \pm 15,0$ & $67 a \pm 41,18$ & $12 c \pm 0,88$ \\
\hline $\mathrm{T} 4-40 \%$ LE $/ 60 \%$ FC & $222 c \pm 9,85$ & $23466 d \pm 2593,4$ & $120 d \pm 10,9$ & $116 a \pm 108,11$ & $19 b \pm 4,71$ \\
\hline T5-20\% LE /80\% FC & $183 c \pm 20,79$ & $19333 d \pm 693,4$ & $112 d \pm 7,8$ & $69 a \pm 46,52$ & $28 \mathrm{a} \pm 3,02$ \\
\hline T6-80\% LE / $20 \%$ VER & $263 b \pm 23,15$ & $28983 b \pm 2967,0$ & $127 d \pm 9,7$ & $78 a \pm 50,81$ & $9 c \pm 1,51$ \\
\hline T7-60\% LE /40\% VER & $262 b \pm 15,06$ & $29500 b \pm 2914,2$ & $132 d \pm 7,9$ & $71 \mathrm{a} \pm 43,74$ & $9 c \pm 1,00$ \\
\hline T8-40\% LE /60\% VER & $269 b \pm 26,53$ & $32116 a \pm 2107,9$ & $140 d \pm 11,4$ & $65 a \pm 42,10$ & $10 c \pm 1,82$ \\
\hline T9-20\% LE /80\% VER & $202 \mathrm{c} \pm 24,16$ & $28816 b \pm 1437,3$ & $134 d \pm 9,5$ & $51 \mathrm{a} \pm 34,21$ & $9 c \pm 1,26$ \\
\hline T10-80\% LE /20\% PCN & $347 a \pm 6,27$ & $34000 \mathrm{a} \pm 998,7$ & $139 d \pm 8,8$ & $86 a \pm 56,11$ & $11 c \pm 2,48$ \\
\hline T11-60\% LE /40\% PCN & $307 a \pm 32,71$ & $33083 a \pm 1304,2$ & $132 d \pm 13,6$ & $81 a \pm 53,04$ & $12 \mathrm{c} \pm 4,99$ \\
\hline $\mathrm{T} 12-40 \%$ LE $/ 60 \%$ PCN & $288 b \pm 15,70$ & $32100 \mathrm{a} \pm 700,0$ & $128 d \pm 10,5$ & $78 a \pm 50,05$ & $13 c \pm 1,61$ \\
\hline T13-20\% LE /80\% PCN & $147 d \pm 10,63$ & 15783 e $\pm 852,0$ & $97 d \pm 0,8$ & $43 a \pm 29,41$ & $29 \mathrm{a} \pm 7,94$ \\
\hline T14-80\% LE /20\% CO & $280 b \pm 12,49$ & $30533 b \pm 768,7$ & $153 d \pm 16,6$ & $72 \mathrm{a} \pm 47,13$ & $10 \mathrm{c} \pm 1,95$ \\
\hline T15-60\% LE /40\% CO & $242 b \pm 19,23$ & $26316 c \pm 750,6$ & $199 c \pm 16,9$ & $52 \mathrm{a} \pm 33,94$ & $14 c \pm 3,33$ \\
\hline T16-40\% LE $/ 60 \%$ CO & $201 c \pm 10,25$ & $19733 d \pm 1537,3$ & $263 b \pm 25,5$ & $38 a \pm 1,45$ & $15 c \pm 6,19$ \\
\hline T17-20\% LE $/ 80 \%$ CO & $127 d \pm 9,51$ & $18766 d \pm 1257,3$ & $282 b \pm 20,6$ & $28 \mathrm{a} \pm 1,18$ & $16 c \pm 4,71$ \\
\hline T18-80\% LE /20\% CAC & $330 a \pm 11,42$ & $26133 c \pm 2139,1$ & $168 \mathrm{c} \pm 12,1$ & $55 a \pm 36,20$ & $12 c \pm 6,92$ \\
\hline T19-60\% LE $/ 40 \%$ CAC & $334 a \pm 60,22$ & $25933 \mathrm{c} \pm 4581,0$ & $181 \mathrm{c} \pm 38,2$ & $56 a \pm 39,31$ & $10 \mathrm{c} \pm 2,80$ \\
\hline T20-40\% LE $/ 60 \%$ CAC & $283 b \pm 15,65$ & $21983 d \pm 832,7$ & $185 c \pm 13,6$ & $51 \mathrm{a} \pm 34,77$ & $10 \mathrm{c} \pm 2,77$ \\
\hline T21-20\% LE /80\% CAC & $251 b \pm 24,25$ & $19733 d \pm 375,3$ & $274 b \pm 18,3$ & $46 a \pm 31,21$ & $12 \mathrm{c} \pm 3,89$ \\
\hline $\mathrm{T} 22-80 \%$ LE $/ 20 \%$ CAN & $360 a \pm 11,59$ & $26900 \mathrm{c} \pm 1105,7$ & $201 c \pm 10,3$ & $62 \mathrm{a} \pm 42,69$ & $9 \mathrm{c} \pm 4,15$ \\
\hline T23-60\% LE $/ 40 \%$ CAN & $311 \mathrm{a} \pm 9,10$ & $24516 c \pm 1926,4$ & $246 b \pm 25,5$ & $48 \mathrm{a} \pm 31,14$ & $9 c \pm 2,05$ \\
\hline T24-40\% LE /60\% CAN & $247 b \pm 10,04$ & 17066 e $\pm 2191,1$ & $282 b \pm 30,4$ & $36 a \pm 23,57$ & $10 \mathrm{c} \pm 2,96$ \\
\hline T25-20\% LE $/ 80 \%$ CAN & $197 c \pm 51,99$ & $12233 \mathrm{f} \pm 1020,2$ & $511 a \pm 29,6$ & $36 a \pm 0,55$ & $11 \mathrm{c} \pm 3,57$ \\
\hline T26-100\% SC & $119 d \pm 34,85$ & $21333 d \pm 5051,3$ & $283 b \pm 27,8$ & $46 a \pm 31,18$ & $24 \mathrm{a} \pm 6,09$ \\
\hline CV $(\%)^{* *}$ & 26,57 & 24,05 & 47,55 & 65,65 & 46,88 \\
\hline
\end{tabular}

LE - Lodo de esgoto; FC - Fibra de coco triturada; VER - Vermiculita; PCN - Palha de café in natura; CO - Composto orgânico; CAC - Casca de arroz carbonizada; CAN - Casca de arroz in natura; $\mathrm{SC}$ - Substrato comercial *Médias seguidas da mesma letra na coluna não diferem estatisticamente entre si pelo teste Scott-Knott ( $p<0,05)$; **Coeficiente de variação 
com maior teor de Zn, 911,63 mg kg-1 e menor de Fe 20515 mg $\mathrm{kg}^{-1}$ em relação ao referido estudo. Os teores totais de $\mathrm{Zn}$ se correlacionaram positivamente com os teores de $\mathrm{N}(\mathrm{r}=0,64) \mathrm{e}$ $\mathrm{P}(\mathrm{r}=0,60)$ e negativamente com o $\mathrm{K}(\mathrm{r}=-0,58)$ enquanto os teores de Fe se correlacionaram de forma positiva com os teores de $\mathrm{Mg}(\mathrm{r}=0,38)$ (Tabela 6).

Os teores totais de $\mathrm{Cu}$ não diferiram estatisticamente entre si nas formulações dos substratos. O substrato comercial, a fibra de coco (60 e $80 \%)$ e a palha de café in natura $(80 \%)$ proporcionaram maior teor de B entre os substratos formulados. Higashikawa et al. (2010) encontraram teores médios de B entre 4,3 (lodo de esgoto) para 31,0 $\mathrm{mg} \mathrm{kg}^{-1}$ (esterco de galinha). Os resíduos com valores intermediários de $\mathrm{B}$ foram palha de café, esterco bovino, fibra de coco e turfa, com valores inferiores a $10 \mathrm{mg} \mathrm{kg}^{-1}$.

No que se refere aos teores totais de Mn e mesmo não havendo grande variação entre as médias dos tratamentos, o composto orgânico, casca de arroz carbonizada e casca de arroz in natura foram responsáveis por elevar o teor total de Mn com a adição desses resíduos. De forma semelhante ao presente estudo, Guerrini \& Trigueiro (2004) verificaram que o aumento do teor total de Mn esteve associado ao aumento da casca de arroz carbonizada e à diminuição do lodo de esgoto.

\section{Conclusões}

1. O lodo de esgoto proporcionou aumento da fertilidade dos substratos com aumento de teores dos nutrientes, principalmente nitrogênio, cálcio e fósforo.

2. De acordo com as características químicas apresentadas apoiando-se na literatura, foram considerados mais adequados para o crescimento de mudas de espécies florestais os substratos formulados com 20 a $80 \%$ de composto orgânico.

3. O lodo de esgoto utilizado nesta pesquisa não promoveu aumento do $\mathrm{pH}$ dos substratos e deve ser utilizado em conjunto com outros resíduos ou ter seu valor de $\mathrm{pH}$ corrigido, de forma a elevá-lo para a faixa ideal visando ao crescimento das mudas.

4. Nenhum substrato testado, inclusive a testemunha, apresentou valores considerados ideais em todas as características químicas avaliadas.

\section{Agradecimentos}

À CAPES, pela concessão da bolsa de estudos, que possibilitou o trabalho.

\section{Literatura Citada}

Araujo, F. F.; Gil, F. C.; Tiritan, C. S. Lodo de esgoto na fertilidade do solo, na nutrição de Brachiaria decumbens e na atividade da desidrogenase. Pesquisa Agropecuária Tropical, v.39, p.1-6, 2009.

Assis, A. M.; Unemoto, L.; Yamamoto, L. Y.; Lone, A. B.; Souza, G. R. B.; Faria, R. T.; Roberto, S. R.; Takahashi, L. S. A. Cultivo de orquídea em substratos à base de casca de café. Bragantia, v.70, p.544-549, 2011.
Backes, C.; Lima, C. P.; Fernandes, D. M.; Godoy, L. G.; Kihl, T. A. M.; Villas Boas, R. L. Efeito do lodo de esgoto e nitrogênio na nutrição e desenvolvimento inicial da mamoneira. Bioscience Journal, v.25, p.90-98, 2009.

Barros, N. F. de; Novais, R. F. de. Eucalipto. In: Ribeiro, A. C.; Guimarães, P. T. G.; Alvarez V.; V. H. (ed.). Recomendações para uso de corretivos e fertilizantes em Minas Gerais: $5^{\mathrm{a}}$ aproximação. Viçosa: Comissão de Fertilidade do Solo do Estado de Minas Gerais, 1999. Cap. 18.4, p.303-305.

Bellote, A. F. J.; Dedecek, R. A. Atributos físicos e químicos do solo e suas relações com o crescimento e a produtividade do Pinus taeda. Boletim de Pesquisa Florestal, v.53, p.2138, 2006.

Brasil. Ministério do Meio Ambiente, Conselho Nacional de Meio Ambiente, CONAMA. Resolução CONAMA $\mathrm{n}^{\circ}$ 375/2006, de 30 de agosto de 2006. Resoluções, 2006. http://www.mma.gov.br/port/conama/legiano1. $\mathrm{cfm}$ ?ano=todos\&codlegitipo=3. 17 Out. 2012.

Cabrera, R. I. Propiedades, uso y manejo de sustratos de cultivo para la producción de plantas en maceta. Revista ChapingoSerie Horticultura, v.1, p.5-11, 1999.

Caldeira, M. V. W.; Rosa, G. N.; Fenilli, T. A. B.; Harbs, R. M. P. Composto orgânico na produção de mudas de aroeiravermelha. Scientia Agraria,v.9, p.27-33. 2008.

Corrêa, R. S.; Silva, L. C. R.; Baptista, G. M. M.; Santos, P. F. dos. Fertilidade química de um substrato tratado com lodo de esgoto e composto de resíduos domésticos. Revista Brasileira de Engenharia Agrícola e Ambiental, v.14, p.538-544, 2010.

Corrêa, R. S.; White, R. E.; Weatherley, A. J. Biosolids effectiveness to yield ryegrass based on their nitrogen content. Scientia Agricola, v.62, p.274-280, 2005.

EMBRAPA - Empresa Brasileira de Pesquisa Agropecuária. Manual de análises químicas de solos, plantas e fertilizantes. Rio de Janeiro: Embrapa informação Tecnológica. 2009. 627p.

Gonçalves, J. L. de M.; Santerelli, E. G.; Moraes Neto, S. P.; Manara, M. P. Produção de mudas de espécies nativas: substrato, nutrição, sombreamento e fertilização. In: Gonçalves, J. L. M.; Benedetti, V. (ed.) Nutrição e fertilização florestal. Piracicaba: ESALQ/USP, 2000.p.309-350.

Gonçalves, L. M.; Poggiani, F. Substratos para produção de mudas florestais. In: Congresso Latino Americano de Ciência Do Solo, 13, 1996. Águas de Lindóia, 1996. Resumos...Piracicaba: Sociedade Latino Americana de Ciência do Solo, 1996. CD-Rom

Guerrini, I. A.; Trigueiro, R. M. Atributos físicos e químicos de substratos compostos por biossólidos e casca de arroz carbonizada. Revista Brasileira de Ciência do Solo, v.28, p.1069-1076, 2004.

Higashikawa, F. S.; Silva, C. A.; Bettiol, W. Chemical and physical properties of organic residues. Revista Brasileira de Ciência do Solo, v.34, p.1742-1752, 2010.

Kämpf, A. N. Produção comercial de plantas ornamentais. Guaíba: Agrolivros, 2.ed, 2005. 254p.

Kratz, D.; Wendling, I.; Nogueira, A. C.; Souza, P. V. de. Propriedades físicas e químicas de substratos renováveis. Revista Árvore, v.37, p.1103-1113, 2013. 
Kratz, D.; Wendling, I.; Pires, P. P. Miniestaquia de Eucalyptus benthamii $\mathrm{x}$ E. dunnii em substratos a base de casca de arroz carbonizada. Scientia Forestalis, v.40, p.547-556, 2012.

MAPA - Ministério da Agricultura, Pecuária e Abastecimento. Instrução Normativa SDA No 17. Diário Oficial da União - Seção 1, n. 99, 24 Maio. 2007. Métodos analíticos oficiais para análise de substratos para plantas e condicionadores de solo. Brasília: MAPA, 2007. 9p.

Oro, T. H.; Quiqui, E. M. del; Oro, P. Crescimento inicial de Eucalyptus grandis Hill ex Maiden tratado com diferentes doses de lodo de esgoto no Noroeste do Paraná. Revista Cultivando o Saber, v.5, p.47-53, 2012.

Paiva, A. V.; Poggiani, F; Goncalves, J. L. M.; Ferraz, A. V. Crescimento de mudas de espécies arbóreas nativas, adubadas com diferentes doses de lodo de esgoto seco e com fertilização mineral. ScientiaForestalis, v.37, p.499$511,2009$.

R Core Team. R: A language and environment for statistical computing. R Foundation for Statistical Computing, Vienna, Austria. 2012. http://www.R-project.org/. 5 Jan. 2013.

Saidelles, F. L. F.; Caldeira, M. V. W.; Schirmer, W. N.; Sperandio, H. V. Casca de arroz carbonizada como substrato para produção de mudas de tamboril-da-mata e garapeira. Semina: Ciências Agrárias, v.30, p.173-1186. 2009.
Schmitz, J. A. K.; Souza, P. V. D.; Kämpf, A. N. Propriedades químicas e físicas de substratos de origem mineral e orgânica para o cultivo de mudas em recipientes. Ciência Rural, v.32, p.937-944, 2002.

Silva, P. H. M. da.; Poggiani, F.; Gonçalves, J. L. de M.; Stape, J. L.; Moreira, R. M. Crescimento de Eucalyptus grandis tratado com diferentes doses de lodos de esgoto úmido e seco, condicionados com polímeros. Scientia Florestalis, v.36, p.79-88, 2008.

Trazzi, P. A.; Caldeira, M. V. W.; Colombi, R.; Gonçalves, E. O. Qualidade de mudas de Murraya paniculata produzidas em diferentes substratos. Floresta, v.42, p.621-630. 2012.

Trigueiro, R. de M.; Guerrini, I. A. Uso de biossólido como substrato para produção de mudas de eucalipto. Scientia Forestalis, v.64, p.150-162, 2003.

Ugarte, J. F. de O.; Sampaio, J. A.; França, S. C. A. Vermiculita. In: Luz, A. B. da; Lins, F. A. F. Rochas \& Minerais Industriais. Rio de Janeiro: CETEM/MCT, 2005. Cap.32, p.677-698.

Vieira, R. F.; Tsai, S. M.; Teixeira, M. A. Efeito do lodo de esgoto no crescimento e fixação simbiotica de N2 em feijoeiro (Phaseolus vulgaris L.). Jaguariúna: Embrapa Meio Ambiente. 2004. 18p. Boletim de Pesquisa e Desenvolvimento

Wendling, I.; Guastala, D; Dedecek, R. Características físicas e químicas de substratos para produção de mudas de Ilex paraguariensis St. Hil. Revista Árvore, v.31, p.209-220, 2007. 